\title{
Comparative analysis of viral RNA and apoptotic cells in bursae following infection with infectious bursal disease virus
}

\begin{abstract}
Specific-pathogen-free (SPF) chickens infected with very virulent (vv) infectious bursal disease virus (IBDV) UPM94/273 developed lower pathogenicity compared to UPM97/61. Sequence analysis indicated that UPM94/273 is an exceptional vvIBDV. In this study, a SYBR ${ }^{\circledR}$ Green I based real-time reverse transcriptase reaction assay was developed to measure viral RNA in the bursae of SPF chickens infected with IBDV. Specificity of the amplified products was confirmed by melting temperature analysis. A linear relationship was observed between the amount of input viral RNA and the threshold values for IBDV-specific product over five $\log 10$ dilutions. The viral RNA level following infection with UPM94/273 was significantly higher at day 1 and 2 post-inoculation (p.i.) compared to UPM97/61 infected chickens. However, chickens infected with UPM97/61 had significantly higher numbers of bursal cells undergoing apoptosis compared to UPM94/273 infected chickens. In both groups, the number of apoptotic cells and viral RNA levels peak at day 3 p.i. This study indicates that UPM97/61 and UPM94/273 have different efficiency of replication and percentage of apoptotic cells in bursae during the acute phase of IBDV infection. (C) 2004 Elsevier Ltd. All rights reserved.
\end{abstract}

Keyword: Apoptosis; Chicken; Infectious bursal disease virus; Real-time RT-PCR 